\title{
Mitochondrion: I am more than a fuel server
}

\author{
Santanu Dasgupta \\ Department of Medicine, The University of Texas Health Science Center at Tyler, Tyler, Texas, USA \\ Correspondence to: Santanu Dasgupta. Department of Medicine, The University of Texas Health Science Center at Tyler, Tyler, Texas, USA. \\ Email: santanu.dasgupta@uthct.edu.
}

\begin{abstract}
Apart from reliable management of the "powerhouse" of the cell, mitochondria faithfully orchestrate a diverse array of important and critical functions in governing cellular signaling, apoptosis, autophagy, mitophagy and innate and adaptive immune system. Introduction of instability and imbalance in the mitochondrial own genome or the nuclear encoded mitochondrial proteome would result in the manifestation of various diseases through alterations in the oxidative phosphorylation system (OXPHOS) and nuclear-mitochondria retrograde signaling. Understanding mitochondrial biology and dynamism are thus of paramount importance to develop strategies to prevent or treat various diseases caused due to mitochondrial alterations.
\end{abstract}

Keywords: Mitochondria; cancer; genetic disorders; therapy; biomarker

Submitted Jul 28, 2019. Accepted for publication Jul 31, 2019.

doi: 10.21037/atm.2019.08.22

View this article at: http://dx.doi.org/10.21037/atm.2019.08.22

\section{Introduction}

Mitochondria are unique cytoplasmic organelles faithfully generating fuel to support healthy living and carrying out normal metabolic function (1). Mitochondria possess their own DNA (mtDNA), strictly follow maternal inheritance and are integral part of the oxidative phosphorylation system (OXPHOS) in producing cellular ATP (1). They are the central regulators of OXPHOS system and constituted of five respiratory complexes (complex I-V) which are assembled from multiple polypeptides, some encoded by mtDNA and others by nuclear DNA (nDNA). The human mtDNA is a $16.5-\mathrm{kb}$ double stranded closed circular molecule, which encodes $12 \mathrm{~S}$ and $16 \mathrm{~S}$ rRNAs, 22 tRNAs and 13 proteins essential for the mitochondrial respiratory complex (1) (Figure 1). Remarkably, intracellular and intercellular mitochondrial heterogeneity exist and each mitochondrion contain hundreds of copies of mtDNA and nearly all of these mtDNA copies remains identical or homoplasmic at birth. Notably, due to the lack of protective histones, rate of mutation in mtDNA is approximately 10 times higher than the nuclear DNA (nDNA) and comparatively easier to spot due to the high copy number particularly in the malignant cells. It is overwhelming to think from the evolutionary perspective about the retention of $13 \mathrm{mtDNA}$ encoded proteins in the mitochondria, while more than 1,500 molecules are traveling from the nucleus to orchestrate diversified mitochondrial function efficiently.

\section{Mitochondria in various cellular signaling}

Appreciable number of studies in the past few decades uncovered diverse functions of mitochondria in regulating inflammation, innate and adaptive immune system, $\mathrm{T}$ cell function, macrophage polarization and mitophagy (2-8). Mitochondria appear to be intimately associated with immune system function by controlling various cellular responses. Inflammation is a natural response to infection or injury to tissues allowing a reasonable amount of time to clear infection or repairing injury. However, a chronic and uncontrolled inflammatory response could induce abnormal changes in the cellular or tissue microenvironment leading to even tumorigenesis (9). In pro-inflammatory microenvironment, mitochondria can elicit unique response known as mitochondrial danger-associated molecular pattern (mtDAMPs) through mtDNA, mtROS, ATP and cardiolipin, calreticulin production, indicative of disruption in mitochondrial homeostasis (10). Importantly, if the 


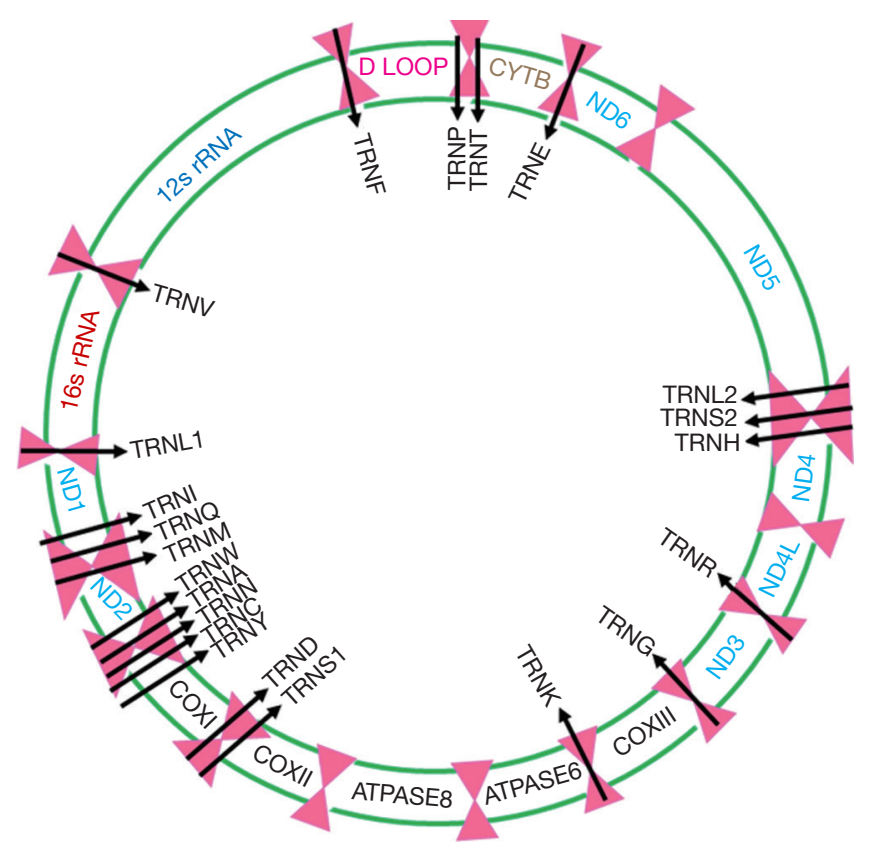

Figure 1 The human mitochondrial genome. The $16.5 \mathrm{~Kb}$ human mtDNA encodes for 13 proteins: CYTB (RCIII), ND6, ND5, ND4, ND4L, ND3, ND2, and ND1 (RCI), COXI, COXII and COXIII (RCIV), ATPASE6 and ATPASE8 (RCV), 12S and $16 \mathrm{~S}$ rRNAs and 22 tRNAs (black arrows) essential for the mitochondrial respiratory complex.

release of mtDAMPs is sustained for a long enough time, it may promote tumorigenic growth and progression (10). However, various mtDAMPs released by the cancer cells can awake and trigger activation of immune cells, such as dendritic cells (DCs), cytotoxic lymphocytes (CTS) or phagocytic macrophages, which can eliminate tumor cells efficiently (10). Among these immune cells, macrophages are the integral components of the innate immune system and mitochondrial metabolism control activation, polarization and inflammatory response generated by the macrophages thereby engaging and directing them towards the mtDAMPs signaling (11). Other than the macrophages, a very recent study from Chandel laboratory has demonstrated a remarkable dependency of regulatory $\mathrm{T}$ cells $\left(\mathrm{T}_{\text {reg }}\right)$ on mitochondrial respiratory complex (RC) III for their classical immune suppressive function (12). The same group also uncovered a novel role of mitochondrial RC III in regulating endothelial cell proliferation during angiogenesis (13). Mitochondria are also potential mediator of anti-viral immune response through mitochondrial antiviral signaling protein (MAVS) (7). Mitophagy is a specialized form of autophagy in which damaged, dysfunctional or obsolete mitochondria are recognized by the autophagy machinery and eventually degraded by the lysosome (14). Mitophagy is regulated by various molecular pathways involving PINK, Parkin, BNIP3, NIX and FUNDC1 (14). Cancer cells can efficiently utilize mitophagy as a survival route and recycle intracellular components when stressed metabolically or exposed to treatment with various anticancer regimens (14).

\section{Mitochondria, heterogeneity, tumorigenesis and health disparity}

Unique existence of intracellular and intercellular mitochondrial heterogeneity exists within cells implicating the enormous complexity of mitochondrial signaling and function in multiple pathways. Figure 2 shows both intracellular and intercellular mitochondrial heterogeneity in human urothelial carcinoma cells analyzed through transmission electron microscopy (15). Mitochondria from 4 different cells exhibit remarkable heterogeneity in their dynamics and distribution. Overwhelmingly, each mitochondrion in the same or different cells in turn may harbor variable copies of both wild type and mutant DNA (a state of heteroplasmy). Supporting this notion, majority of the mtDNA deep sequencing studies including ours have reported the presence of heteroplasmic mtDNA mutations (15-18).

A fundamental role of mitochondrial alterations in cancer evolution has been enunciated long ago by Otto Warburg $(19,20)$. That obscured path has been uncovered recently, which continues to provide solid evidence that mtDNA mutations and changes in mitochondrial dynamics substantially contribute to tumorigenesis (21-28). To date, mitochondrial DNA mutation encompassing the coding and non-coding regions of the mitochondria have been detected in human malignancies of various anatomic origin (1,15-17,29-40). As mentioned above, most of these mutations were heteroplasmic in nature (1). Colorectal cancer (CRC) was the first studied neoplasm for mtDNA mutation and majority of the CRC mutations were centered in the non-coding tRNA and rRNA genes (41-44). In breast cancer (BC), the most frequently altered mtDNA regions were ATP6, ATP8 (RCV) and ND5 (RCI) (45-48). Recent studies in gastric carcinomas (GC) identified several mtDNA mutations encompassing the regulatory D-loop 

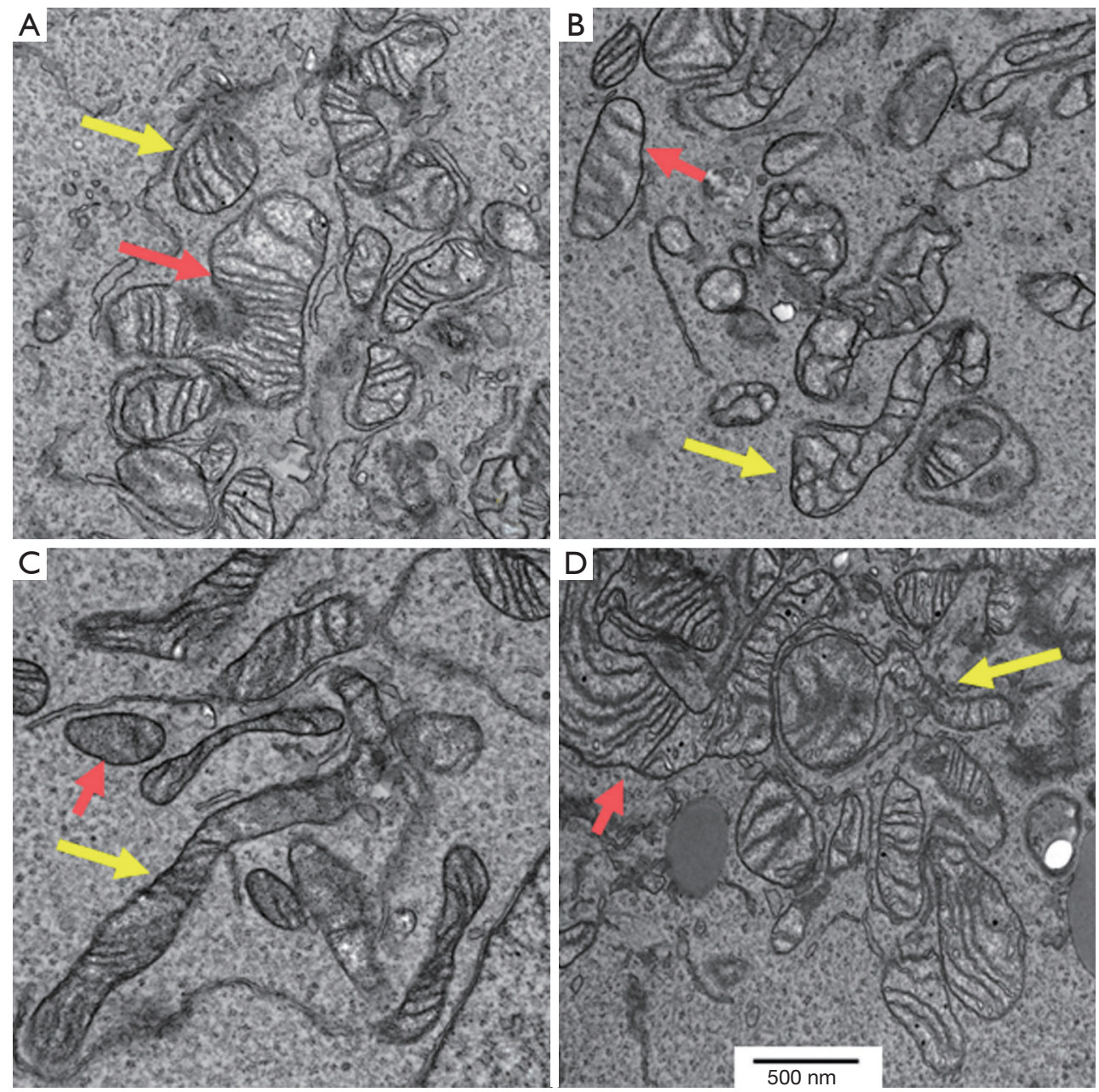

Figure 2 Mitochondrial heterogeneity. Transmission electron micrographs showing intracellular and intercellular mitochondrial heterogeneity (yellow vs. red arrows) in term of dynamics and distribution pattern in four different (A-D) urothelial carcinoma cells. Different cells were selected in a random fashion for the imaging. Magnification $\times 30,000$.

and tRNA regions. On the other hand, non-coding rRNA, tRNA accompanied with both germ line and somatic mtDNA mutation from the respiratory complex (RC)-I, RCIV, RCIII regions were detected in head and neck cancer (49-52). Similarly in lung cancer, RCI and non-coding tRNA and rRNA mtDNA mutations were detected (53-57). Several groups have examined the extent of mtDNA mutations in prostate cancer and detected predominant mtDNA mutations in RCIV region along with non-coding mutations in rRNA and tRNA genes (39,58-62). A recent study identified RCI mutations exclusively in bone metastatic tissues from recurrent prostate cancer patients (18). In urothelial cell cancer, mtDNA mutations in protein-coding RCI (ND3, ND4, ND5) and RCIII (CYTB) have been reported (17,63-65). The human papilloma virus associated cervical cancer (CC) has also been studied and detected mtDNA mutations in D-loop, tRNA and rRNA genes and RCI (ND5) region (66-68). In addition to CC, mtDNA D-loop sequence variants were also detected in cervical intraepithelial neoplastic tissues suggesting a possible role of mtDNA alterations in early neoplastic transformation (68). The hurthle cell carcinoma develops from thyroid follicular cells, highly invasive in nature and harbor unique abundance of dysfunctional mitochondria (69). Higher abundance of mtDNA mutation ( $>2$ mutations per positive samples) was detected in $24 \%(17 / 49)$ hurthle cell carcinoma subjects. The mutations were from various $\mathrm{mtDNA}$ regions including tRNA and coding RCI (ND6) and RCV (69) 
(ATP8). Another interesting study identified RCI mutation as potential driver of hurthle cell carcinomas, where a total of 29 protein coding and 17 non-coding mtDNA mutations were detected (36). Although, numerous studies described above have identified non-coding (D-loop, tRNA, rRNA) and coding (RCI, III, IV and V) mtDNA mutations, only a limited number of studies have demonstrated their functional role in tumorigenesis.

In addition to mtDNA sequence variants, alteration in mtDNA copy number reflecting mitochondrial dysfunction has also been detected in various cancers including bladder, colorectal, gastric, head and neck, lung and prostate cancer $(1,24,53,54,64,70)$. Functional studies with patient derived mtDNA mutations by us and others also revealed tumor growth and metastasis promoting and immune evasive role of mtDNA mutations confirming the contribution of mitochondrial alterations in tumorigenesis $(53,62,65,71,72)$. On the other hand, tumor-derived clonal mtDNA mutations detected in urine, histopathologically normal surgical margins and salivary rinse samples from the same patients indicates their potential in biomarker development $(30,51,64)$. Additionally, correlation between cancer promoting key nuclear genes such as EGFR, PSA and mtDNA mutations has also been established in lung and prostate cancer respectively $(31,73)$. However, the fundamental and causative role of patients' derived mtDNA mutations in cancer initiation being within a heteroplasmic environment and their utilization in early cancer detection, monitoring and surveillance is yet to be established. Deciphering these critical contributions of mitochondria could open up novel avenues for therapeutic and biomarker development in clinical settings.

Cancer health disparity is a significant problem in various ethnic populations $(74,75)$. Incidences of prostate, head and neck, uterine cervix and breast cancers are significantly higher among the African American with more aggressive outcomes and poor survival compared to the Caucasian American Population (74-78). Moreover, CC appears to be higher and more aggressive among the Hispanic/Latina women compared to the African American and Caucasian American women (78). The mitochondrial biological basis of this disparate outcome is yet to be established. In recent studies, an association between G10398A sequence variant in mitochondria encoded RCI (ND3, Figure 1) and increased risk of breast and prostate cancer among the African American subjects was reported (74). Among the Caucasian, detection of U haplogroup marker A12308G
$\left(\mathrm{tRNA}^{\text {leu2}}\right)$ appeared to be associated with increased risk of prostate and renal cancer (74). In addition, co-detection of T4216C (RCI-ND1, Figure 1) and G10398 (RCIND3, Figure 1) appear to be associated with increase breast cancer risk among the African American women (74). Other than mitochondrial DNA (mtDNA) sequence variants, alterations in mtDNA copy numbers were found to be associated with cancer health disparities among the African American subjects including prostate cancer $(74,79)$. However, a comprehensive analysis delineating the precise functional role of mtDNA alterations (both mutation and copy number changes) in cancer health disparities has yet to be done.

\section{Mitochondrial alteration in genetic disease}

Other than cancer, mtDNA alterations have been linked to various genetic diseases including mitochondrial encephalopathy, lactic acidosis, and stroke-like episodes (MELAS), Leber's hereditary optic neuropathy (LHON), Leigh syndrome (LS), mitochondrial encephalopathy, Kearns-Sayre syndrome (KSS), progressive external ophthalmoplegia (PEO), Parkinson's, Alzheimer's, Huntington's disease, myopathy, cardiomyopathy, hepatopathy, hearing loss and gastrointestinal dysmotility (80-91). In addition to the mtDNA encoded genes, various nuclear encoded genes that function in mitochondria are linked with many of these diseases and involve $P O L G$, POLG2, TWINK, SLC25A4, TYMP, MPV17, OPA1, MFN2, DGUOK, RRM2B, TK2TFAM, PARKIN, and PINK1 (92). Unfortunately, majority of these diseases are not curable at this time. However, a recent study employing an adenovirus-associated gene delivery of mitochondria targeted zinc-finger nucleases (mtZFN), induced specific elimination of mutant mtDNA across the heart in mice bearing heteroplasmic mtDNA mutation $\mathrm{m} .5024 \mathrm{C}>\mathrm{T}$ $\mathrm{tRNA}^{\mathrm{Ala}}$, causing a cardiac disease (93). This finding raises our hope for a new therapeutic avenue for effectively treating heteroplasmic mitochondrial diseases of diverse genetic origin (93).

\section{Mitochondrial reactive oxygen species (ROS) and oncogenesis}

Mitochondria are the major resources of ROS production during their metabolic function (94). Under normal condition, mitochondrial oxidant scavenging system 
containing the dimutase, catalase and glutathione oxidase such as SOD2, Grx2, Trx, TrxR counterbalance ROS generation $(9,94,95)$. However, impairment of mitochondrial function under various pathological conditions such as neoplastic transformation may sustain ROS production, with concomitant imbalance or loss of function of the ROS scavenging molecules such as SOD2 (9,94-96). In such conditions, abundant ROS generation could positively regulate diverse oncogenic signaling

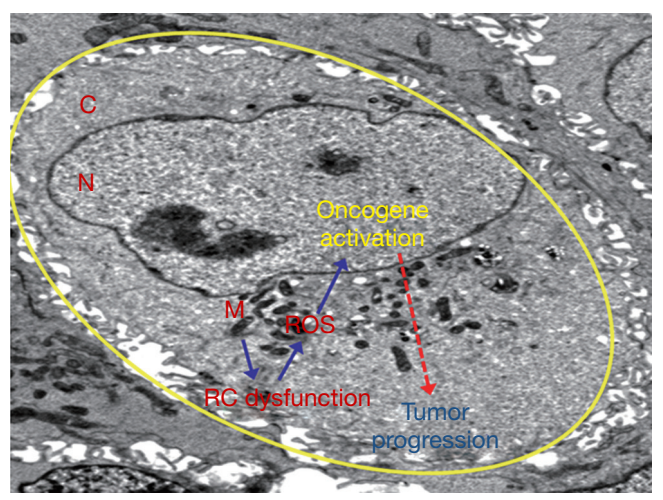

Figure 3 Mitochondria in oncogenesis. Mitochondria (M) harboring heterogeneous mtDNA mutations could induce respiratory complex (RC) dysfunction, which in turn promotes reactive oxygen species (ROS) production and subsequent activation of oncogenic pathways leading to tumorigenic progression. Transmission electron micrograph of mouse urothelial carcinoma cell (MB49) was utilized for mechanistic depiction. Magnification $\times 30,000$. C: cytoplasm; N: Nucleus. network, inhibit the function of various tumor suppressor genes and promote cancer progression (65,95,97-100) (Figure 3).

\section{Conclusion and future perspective}

Mitochondria have been able to attract considerable attention in this decade which continues to unravel their dynamic role in cell growth and signaling, immune modulation, neoplasia and various genetic diseases (Figure 4). However, the existence of enormous intracellular and intercellular mitochondrial as well as mtDNA heterogeneity in concert with complex nDNA-mtDNA signaling are enigmatic and pose significant challenges in designing appropriate treatment for different mitochondrial diseases. However, various models including transgenic animals, cybrids, allotropic mtDNA expression strategies and mitochondria targeted therapeutics are being developed with a view to study and correct these mitochondrial defects $(89,90)$. Moreover, due to the occurrence of frequent RC specific coding and non-coding mtDNA mutations in various cancers and changes in mtDNA content, mtDNA alterations could be useful in developing mitochondria based novel molecular markers. With the advent of cutting age technologies for mitochondria genome sequencing and studying various aspect of mitochondria biology, the next decade would certainly advance our knowledge in the field of mitochondrial genomics and biology and will aid to develop better mitochondrial disease management strategies.

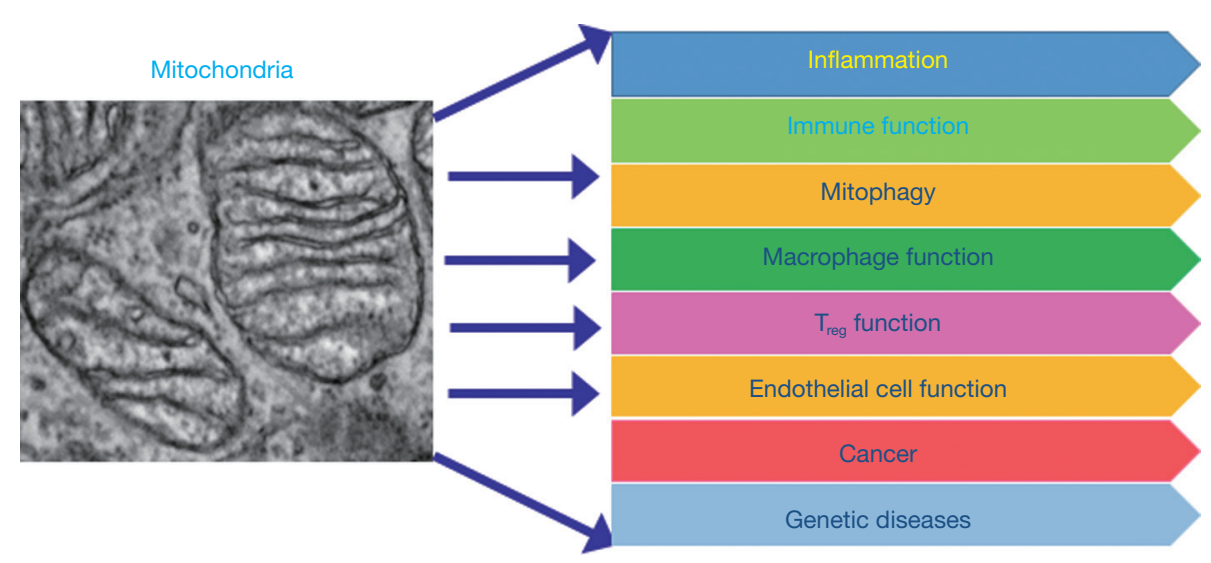

Figure 4 Dynamic role of mitochondria. Versatile functions of mitochondria in governing numerous important cellular processes. Alteration in mitochondrial homeostasis and function may lead to the development of cancer and various genetic diseases. Transmission electron micrograph of human urothelial carcinoma cell was utilized for describing mitochondrial function. Magnification $\times 30,000$. 


\section{Acknowledgments}

The study is supported by the University of Texas Health Science Center at Tyler.

\section{Footnote}

Conflicts of Interest: The author has no conflicts of interest to declare.

Ethical Statement: The author is accountable for all aspects of the work in ensuring that questions related to the accuracy or integrity of any part of the work are appropriately investigated and resolved.

\section{References}

1. Hertweck KL, Dasgupta S. The Landscape of mtDNA Modifications in Cancer: A Tale of Two Cities. Front Oncol 2017;7:262.

2. Tait SW, Green DR. Mitochondria and cell signalling. J Cell Sci 2012;125:807-15.

3. Weinberg SE, Sena LA, Chandel NS. Mitochondria in the regulation of innate and adaptive immunity. Immunity 2015;42:406-17.

4. Chen Y, Zhou Z, Min W. Mitochondria, Oxidative Stress and Innate Immunity. Front Physiol 2018;9:1487.

5. Meyer A, Laverny G, Bernardi L, et al. Mitochondria: An Organelle of Bacterial Origin Controlling Inflammation. Front Immunol 2018;9:536.

6. Dela Cruz CS, Kang MJ. Mitochondrial dysfunction and damage associated molecular patterns (DAMPs) in chronic inflammatory diseases. Mitochondrion 2018;41:37-44.

7. Mohanty A, Tiwari-Pandey R, Pandey NR. Mitochondria: the indispensable players in innate immunity and guardians of the inflammatory response. J Cell Commun Signal 2019. [Epub ahead of print].

8. Chandel NS. Evolution of Mitochondria as Signaling Organelles. Cell Metab 2015;22:204-6.

9. Neagu M CC, Popescu ID, Zipeto D, et al. Inflammation and Metabolism in Cancer Cell-Mitochondria Key Player. Front Oncol 2019;9:348.

10. Kopecka J, Gazzano E, Castella B, et al. Mitochondrial metabolism: Inducer or therapeutic target in tumor immune-resistance? Semin Cell Dev Biol 2019. [Epub ahead of print].

11. Liu PS, Ho PC. Mitochondria: A master regulator in macrophage and $\mathrm{T}$ cell immunity. Mitochondrion
2018;41:45-50.

12. Weinberg SE, Singer BD, Steinert EM, et al. Mitochondrial complex III is essential for suppressive function of regulatory T cells. Nature 2019;565:495-9.

13. Diebold LP, Gil HJ, Gao P, et al. Mitochondrial complex III is necessary for endothelial cell proliferation during angiogenesis. Nat Metab 2019;1:158-71.

14. Ferro F, Servais S, Besson P, et al. Autophagy and mitophagy in cancer metabolic remodelling. Semin Cell Dev Biol 2019. [Epub ahead of print].

15. Philley JV, Kannan A, Qin W, et al. Complex-I Alteration and Enhanced Mitochondrial Fusion Are Associated With Prostate Cancer Progression. J Cell Physiol 2016;231:1364-74.

16. Dasgupta $\mathrm{S}$. The Landscape of mtDNA Modifications in Cancer: A Tale of Two Cities. Sci Rep 2017;7:262.

17. Duberow DP, Brait M, Hoque MO, et al. Highperformance detection of somatic D-loop mutation in urothelial cell carcinoma patients by polymorphism ratio sequencing. J Mol Med (Berl) 2016;94:1015-24.

18. Arnold RS, Fedewa SA, Goodman M, et al. Bone metastasis in prostate cancer: Recurring mitochondrial DNA mutation reveals selective pressure exerted by the bone microenvironment. Bone 2015;78:81-6.

19. Warburg O, Wind F, Negelein E. The metabolism of tumors in the body. J Gen Physiol 1927;8:519-30.

20. Warburg O. On the origin of cancer cells. Science 1956;123:309-14.

21. Weinberg SE, Chandel NS. Targeting mitochondria metabolism for cancer therapy. Nat Chem Biol 2015;11:9-15.

22. Pustylnikov S, Costabile F, Beghi S, et al. Targeting mitochondria in cancer: current concepts and immunotherapy approaches. Transl Res 2018;202:35-51.

23. Leone G, Abla H, Gasparre G, et al. The Oncojanus Paradigm of Respiratory Complex I. Genes (Basel) 2018;9. doi: 10.3390/genes 9050243 .

24. Chen J, Zhang L, Yu X, et al. Clinical application of plasma mitochondrial DNA content in patients with lung cancer. Oncol Lett 2018;16:7074-81.

25. Weerts MJ, Smid M, Foekens JA, et al. Mitochondrial RNA Expression and Single Nucleotide Variants in Association with Clinical Parameters in Primary Breast Cancers. Cancers (Basel) 2018;10. doi: 10.3390/ cancers 10120500.

26. Hahn A, Zuryn S. The Cellular Mitochondrial Genome Landscape in Disease. Trends Cell Biol 2019;29:227-40.

27. Herst PM, Grasso C, Berridge MV. Metabolic 
reprogramming of mitochondrial respiration in metastatic cancer. Cancer Metastasis Rev 2018;37:643-53.

28. Badrinath N, Yoo SY. Mitochondria in cancer: in the aspects of tumorigenesis and targeted therapy. Carcinogenesis 2018;39:1419-30.

29. Sidransky D. Mitochondrial cytochrome B gene mutation promotes tumor growth in bladder cancer. Oncogene 2008;68:700-6.

30. Sidransky D. Following mitochondrial footprints through a long mucosal path to lung cancer. Int J Cancer 2009;4:e6533.

31. Sidransky D. Mitochondrial DNA mutations in respiratory complex-I in never-smoker lung cancer patients contribute to lung cancer progression and associated with EGFR gene mutation. Int J Cancer 2012;227:2451-60.

32. Hsu CC, Lee HC, Wei YH. Mitochondrial DNA alterations and mitochondrial dysfunction in the progression of hepatocellular carcinoma. World J Gastroenterol 2013;19:8880-6.

33. McGeehan RE, Cockram LA, Littlewood DTJ, et al. Deep sequencing reveals the mitochondrial DNA variation landscapes of breast-to-brain metastasis blood samples. Mitochondrial DNA A DNA Mapp Seq Anal 2018;29:703-13.

34. Jarviaho T, Hurme-Niiranen A, Soini HK, et al. Novel non-neutral mitochondrial DNA mutations found in childhood acute lymphoblastic leukemia. Clin Genet 2018;93:275-85.

35. Palodhi A, Ghosh S, Biswas NK, et al. Profiling of genomic alterations of mitochondrial DNA in gingivobuccal oral squamous cell carcinoma: Implications for disease progress. Mitochondrion 2019;46:361-9.

36. Gopal RK, Kubler K, Calvo SE, et al. Widespread Chromosomal Losses and Mitochondrial DNA Alterations as Genetic Drivers in Hurthle Cell Carcinoma. Cancer Cell 2018;34:242-55.e5.

37. Zeng AG, Leung AC, Brooks-Wilson AR. Somatic Mitochondrial DNA Mutations in Diffuse Large B-Cell Lymphoma. Sci Rep 2018;8:3623.

38. Hopkins JF, Denroche RE, Aguiar JA, et al. Mutations in Mitochondrial DNA From Pancreatic Ductal Adenocarcinomas Associate With Survival Times of Patients and Accumulate as Tumors Progress. Gastroenterology 2018;154:1620-4.e5.

39. Arnold RS, Sun Q, Sun CQ, et al. An inherited heteroplasmic mutation in mitochondrial gene $\mathrm{COI}$ in a patient with prostate cancer alters reactive oxygen, reactive nitrogen and proliferation. Biomed Res Int
2013;2013:239257.

40. Triska P, Kaneva K, Merkurjev D, et al. Landscape of germline and somatic mitochondrial DNA mutations in pediatric malignancies. Cancer Res 2019;79:1318-30.

41. Adams G Jr, Mehrabi S, Vatcharapijarn Y, et al. Frequencies of mtDNA mutations in primary tissues of colorectal adenopolyps. Front Biosci (Elite Ed) 2013;5:809-13.

42. de Araujo LF, Fonseca AS, Muys BR, et al. Mitochondrial genome instability in colorectal adenoma and adenocarcinoma. Tumour Biol 2015;36:8869-79.

43. Errichiello E, Venesio T. Mitochondrial DNA variants in colorectal carcinogenesis: Drivers or passengers? J Cancer Res Clin Oncol 2017;143:1905-14.

44. Kleist B, Meurer T, Poetsch M. Mitochondrial DNA alteration in primary and metastatic colorectal cancer: Different frequency and association with selected clinicopathological and molecular markers. Tumour Biol 2017;39:1010428317692246.

45. Alhomidi MA, Vedicherla B, Movva S, et al. Mitochondrial D310 instability in Asian Indian breast cancer patients. Tumour Biol 2013;34:2427-32.

46. Grzybowska-Szatkowska L, Slaska B, Rzymowska J, et al. Novel mitochondrial mutations in the ATP6 and ATP8 genes in patients with breast cancer. Mol Med Rep 2014;10:1772-8.

47. Meng XL, Meng H, Zhang W, et al. The role of mitochondrial tRNA variants in female breast cancer. Mitochondrial DNA A DNA Mapp Seq Anal 2016;27:3199-201.

48. Yadav N, Chandra D. Mitochondrial DNA mutations and breast tumorigenesis. Biochim Biophys Acta 2013;1836:336-44.

49. Guo W, Yang D, Xu H, et al. Mutations in the D-loop region and increased copy number of mitochondrial DNA in human laryngeal squamous cell carcinoma. Mol Biol Rep 2013;40:13-20.

50. Shu HY, Li HC, Xie WQ, et al. Mitochondrial DNA variations in tongue squamous cell carcinoma. Biomed Rep 2019;10:23-8

51. Sidransky D. Mitochondrial DNA mutation in normal margins and tumors of recurrent head and neck squamous cell carcinoma patients. J Thorac Oncol 2010;3:1205-11.

52. Liu SA, Jiang RS, Wang WY, et al. Somatic mutations in the D-loop of mitochondrial DNA in head and neck squamous cell carcinoma. Head Neck 2015;37:878-83.

53. Dasgupta S, Soudry E, Mukhopadhyay N, et al. Mitochondrial DNA mutations in respiratory complex-I 
in never-smoker lung cancer patients contribute to lung cancer progression and associated with EGFR gene mutation. J Cell Physiol 2012;227:2451-60.

54. Dasgupta S, Yung RC, Westra WH, et al. Following mitochondrial footprints through a long mucosal path to lung cancer. PLoS One 2009; 4:e6533.

55. Fang Y, Yang HY, Shi YH, et al. Mitochondrial DNA haplogroups and somatic mutations are associated with lung cancer in patients from Southwest China. Genet Mol Res 2015;14:5031-43.

56. Liu F, Sanin DE, Wang X. Mitochondrial DNA in Lung Cancer. Adv Exp Med Biol 2017;1038:9-22.

57. Amer W, Toth C, Vassella E, et al. Evolution analysis of heterogeneous non-small cell lung carcinoma by ultradeep sequencing of the mitochondrial genome. Sci Rep 2017;7:11069.

58. Dasgupta S. Complex-I Alteration and Enhanced Mitochondrial Fusion Are Associated With Prostate Cancer Progression. Clin Cancer Res 2016;231:1364-74.

59. Kalsbeek AM, Chan EF, Grogan J, et al. Mutational load of the mitochondrial genome predicts pathological features and biochemical recurrence in prostate cancer. Aging (Albany NY) 2016;8:2702-12.

60. Lindberg J, Mills IG, Klevebring D, et al. The mitochondrial and autosomal mutation landscapes of prostate cancer. Eur Urol 2013;63:702-8.

61. Hopkins JF, Sabelnykova VY, Weischenfeldt J, et al. Mitochondrial mutations drive prostate cancer aggression. Nat Commun 2017;8:656.

62. Petros JA, Baumann AK, Ruiz-Pesini E, et al. mtDNA mutations increase tumorigenicity in prostate cancer. Proc Natl Acad Sci U S A 2005;102:719-24.

63. Chatterjee A, Dasgupta S, Sidransky D. Mitochondrial subversion in cancer. Cancer Prev Res (Phila) 2011;4:638-54.

64. Dasgupta S, Shao C, Keane TE, et al. Detection of mitochondrial deoxyribonucleic acid alterations in urine from urothelial cell carcinoma patients. Int J Cancer 2012;131:158-64.

65. Dasgupta S, Hoque MO, Upadhyay S, et al. Mitochondrial cytochrome $\mathrm{B}$ gene mutation promotes tumor growth in bladder cancer. Cancer Res 2008;68:700-6.

66. Kabekkodu SP, Bhat S, Mascarenhas R, et al. Mitochondrial DNA variation analysis in cervical cancer. Mitochondrion 2014;16:73-82.

67. Warowicka A, Kwasniewska A, Gozdzicka-Jozefiak A. Alterations in mtDNA: a qualitative and quantitative study associated with cervical cancer development. Gynecol
Oncol 2013;129:193-8.

68. Goia-Rusanu CD, Iancu IV, Botezatu A, et al. Mitochondrial DNA mutations in patients with HRHPVrelated cervical lesions. Roum Arch Microbiol Immunol 2011;70:5-10.

69. Xu B, Reznik E, Tuttle RM, et al. Outcome and molecular characteristics of non-invasive encapsulated follicular variant of papillary thyroid carcinoma with oncocytic features. Endocrine 2019;64:97-108.

70. Reznik E, Miller ML, Senbabaoglu Y, et al. Mitochondrial DNA copy number variation across human cancers. Elife 2016;5. doi: 10.7554/eLife.10769.

71. Ishikawa K, Imanishi H, Takenaga K, et al. Regulation of metastasis; mitochondrial DNA mutations have appeared on stage. J Bioenerg Biomembr 2012;44:639-44.

72. Chen EI. Mitochondrial dysfunction and cancer metastasis. J Bioenerg Biomembr 2012;44:619-22.

73. Kloss-Brandstatter A, Schafer G, Erhart G, et al. Somatic mutations throughout the entire mitochondrial genome are associated with elevated PSA levels in prostate cancer patients. Am J Hum Genet 2010;87:802-12.

74. Choudhury AR, Singh KK. Mitochondrial determinants of cancer health disparities. Semin Cancer Biol 2017;47:125-46.

75. Deshmukh SK, Azim S, Ahmad A, et al. Biological basis of cancer health disparities: resources and challenges for research. Am J Cancer Res 2017;7:1-12.

76. Daraei P, Moore CE. Racial Disparity Among the Head and Neck Cancer Population. J Cancer Educ 2015;30:546-51.

77. Moore CE, Warren R, Maclin SD Jr. Head and neck cancer disparity in underserved communities: probable causes and the ethics involved. J Health Care Poor Underserved 2012;23:88-103.

78. Olusola P, Banerjee HN, Philley JV, et al. Human Papilloma Virus-Associated Cervical Cancer and Health Disparities. Cells 2019;8. doi: 10.3390/cells8060622.

79. Koochekpour S, Marlowe T, Singh KK, et al. Reduced mitochondrial DNA content associates with poor prognosis of prostate cancer in African American men. PLoS One 2013;8:e74688.

80. Carelli V, La Morgia C. Clinical syndromes associated with mtDNA mutations: where we stand after 30 years. Essays Biochem 2018;62:235-54.

81. Franco-Iborra S, Vila M, Perier C. Mitochondrial Quality Control in Neurodegenerative Diseases: Focus on Parkinson's Disease and Huntington's Disease. Front Neurosci 2018;12:342.

82. Salazar C, Ruiz-Hincapie P, Ruiz LM. The Interplay 
among PINK1/PARKIN/Dj-1 Network during

Mitochondrial Quality Control in Cancer Biology:

Protein Interaction Analysis. Cells 2018;7. doi: 10.3390/ cells7100154.

83. Valero T. Mitochondrial biogenesis: pharmacological approaches. Curr Pharm Des 2014;20:5507-9.

84. Mordaunt DA, McIntyre LC, Salvemini H, et al. Presentation of m.3243A>G (MT-TL1; tRNALeu) variant with focal neurology in infancy. Am J Med Genet A 2015;167a:2697-701.

85. Hashimoto M, Bacman SR, Peralta S, et al. MitoTALEN: A General Approach to Reduce Mutant mtDNA Loads and Restore Oxidative Phosphorylation Function in Mitochondrial Diseases. Mol Ther 2015;23:1592-9.

86. Liu F, Zhang D, Yan C. Expanding the clinical spectrum of myopathy, encephalopathy, lactic acidosis, and strokelike (MELAS) episode: A case with A3243G mitochondrial DNA mutation presenting as MELAS and congenital melanocytic naevi overlap. Neurol India 2016;64:336-8.

87. Rajakulendran S, Pitceathly RD, Taanman JW, et al. A Clinical, Neuropathological and Genetic Study of Homozygous A467T POLG-Related Mitochondrial Disease. PLoS One 2016;11:e0145500.

88. Su T, Grady JP, Afshar S, et al. Inherited pathogenic mitochondrial DNA mutations and gastrointestinal stem cell populations. J Pathol 2018;246:427-32.

89. Wallace DC. Mitochondrial genetic medicine. Nat Genet 2018;50:1642-9.

90. Picard M, Wallace DC, Burelle Y. The rise of mitochondria in medicine. Mitochondrion 2016;30:105-16.

91. Coskun P, Wyrembak J, Schriner SE, et al. A mitochondrial etiology of Alzheimer and Parkinson disease. Biochim Biophys Acta 2012;1820:553-64.

Cite this article as: Dasgupta S. Mitochondrion: I am more than a fuel server. Ann Transl Med 2019;7(20):594. doi: 10.21037/ atm.2019.08.22
92. El-Hattab AW, Craigen WJ, Scaglia F. Mitochondrial DNA maintenance defects. Biochim Biophys Acta Mol Basis Dis 2017;1863:1539-55.

93. Gammage PA, Viscomi C, Simard ML, et al. Genome editing in mitochondria corrects a pathogenic mtDNA mutation in vivo. Nat Med 2018;24:1691-5.

94. Moradi-Marjaneh R, Hassanian SM, Mehramiz M, et al. Reactive oxygen species in colorectal cancer: The therapeutic impact and its potential roles in tumor progression via perturbation of cellular and physiological dysregulated pathways. J Cell Physiol 2019;234:10072-9.

95. Idelchik MDPS, Begley U, Begley TJ, et al. Mitochondrial ROS control of cancer. Semin Cancer Biol 2017;47:57-66.

96. Borrelli A, Schiattarella A, Bonelli P, et al. The functional role of MnSOD as a biomarker of human diseases and therapeutic potential of a new isoform of a human recombinant MnSOD. Biomed Res Int 2014;2014:476789.

97. Cui Q, Wang JQ, Assaraf YG, et al. Modulating ROS to overcome multidrug resistance in cancer. Drug Resist Updat 2018;41:1-25.

98. Dasgupta S, Hoque MO, Upadhyay S, et al. Forced cytochrome B gene mutation expression induces mitochondrial proliferation and prevents apoptosis in human uroepithelial SV-HUC-1 cells. Int J Cancer 2009;125:2829-35.

99. Galadari S, Rahman A, Pallichankandy S, et al. Reactive oxygen species and cancer paradox: To promote or to suppress? Free Radic Biol Med 2017;104:144-64.

100.Jezek J, Cooper KF, Strich R. Reactive Oxygen Species and Mitochondrial Dynamics: The Yin and Yang of Mitochondrial Dysfunction and Cancer Progression. Antioxidants (Basel) 2018;7. doi: 10.3390/antiox7010013. 